\title{
Wind loads on ground-based telescopes
}

\author{
Douglas G. MacMynowski, Konstantinos Vogiatzis, George Z. Angeli, Joeleff Fitzsimmons, \\ and Jerry E. Nelson
}

\begin{abstract}
One of the factors that can influence the performance of large optical telescopes is the vibration of the telescope structure due to unsteady wind inside the telescope enclosure. Estimating the resulting degradation in image quality has been difficult because of the relatively poor understanding of the flow characteristics. Significant progress has recently been made, informed by measurements in existing observatories, wind-tunnel tests, and computational fluid dynamic analyses. We combine the information from these sources to summarize the relevant wind characteristics and enable a model of the dynamic wind loads on a telescope structure within an enclosure. The amplitude, temporal spectrum, and spatial distribution of wind disturbances are defined as a function of relevant design parameters, providing a significant improvement in our understanding of an important design issue. () 2006 Optical Society of America

OCIS codes: $110.6770,120.7280$.
\end{abstract}

\section{Introduction}

Design studies are underway for the next generation of large ground-based optical telescopes. While the enclosure surrounding the telescope provides a significant reduction in wind speeds, the residual wind loads that result from large-scale flow structures and turbulence inside the enclosure may still lead to significant vibration.1,2 Estimates of the wind loads are required early in the design process, influencing the design of the enclosure, telescope structure, and control system. The wind parameterization herein reflects our best current understanding of the wind environment inside a roughly hemispherical telescope enclosure, informed by three separate sources of data. Full-scale data are available from measurements taken at Gemini South Observatory. ${ }^{3-5}$ More extensive data under more controlled conditions have been collected in an $\sim 1 \%$ scale

D. G. MacMynowski (macmardg@cds.caltech.edu) is with the California Institute of Technology, 1200 E. California Boulevard, M/C 104-44 Pasadena; California 91125. K. Vogiatzis is with the New Initiatives Office, Association of Universities for Research in Astronomy, NOAO 950 N. Cherry Avenue, Tucson, Arizona 85719. G. Z. Angeli is with the Thirty Meter Telescope Project, 1200 E. California Boulevard, M/C 102-8 Pasadena, California 91125. J. Fitzsimmons is with the Hertzberg Institute for Astrophysics, NRC, 5071 W. Saanich Road, Victoria, British Columbia V9E-2E7, Canada. J. E. Nelson is with the University of California, Santa Cruz, 1156 High Street, Santa Cruz, California 95064.

Received 14 October 2005; revised 22 May 2006; accepted 1 June 2006; posted 6 June 2006 (Doc. ID 65355).

$0003-6935 / 06 / 307912-12 \$ 15.00 / 0$

(C) 2006 Optical Society of America wind-tunnel experiment. ${ }^{6}$ Finally, computational fluid dynamic (CFD) analyses ${ }^{7}$ have been validated against these data sources and used primarily to understand differences between different sources of data.

Understanding the wind inside a telescope enclosure has been of concern for many years. While fullscale tests are ideal, limited data are available both because of the expense and because of the difficulty in controlling test conditions. The pressure was measured at a few points inside the Multiple Mirror Telescope (MMT) dome ${ }^{8}$ the torque on the main drives measured for the Very Large Telescope (VLT), ${ }^{9}$ while recent measurements at the Keck Observatory include simultaneous velocity and torque data. ${ }^{10}$ In contrast, the Gemini data ${ }^{3}$ used herein include 32 pressure measurements over the primary mirror and multiple velocity measurements taken under a variety of conditions. Only flow visualization or limited data at a few points inside the telescope enclosure is available from wind-tunnel tests for Keck ${ }^{11}$ and the VLT. ${ }^{12}$ Significantly more data have been collected in recent tests, leading to both better understanding of the flow field and quantitative information that can be used in modeling and design. Digital particle image velocimetry (DPIV) can provide a quantitative spatial map of the flow field in the region around the secondary mirror, ${ }^{6}$ while arrays of pressure measurements across the primary mirror can be used to understand the spatial variation. ${ }^{13}$ In addition to the wind-tunnel tests on hemispherical domes that are used to develop the understanding herein, others have recently been conducted for a different dome geometry. ${ }^{14}$ Tests have also been conducted for radio 
telescopes; however, since these typically do not have enclosures to shield them from the wind, the flow field around the telescope is quite different. Because of the time-varying three-dimensional nature of the flow, CFD simulations are computationally intensive and have only recently been of value for estimating unsteady wind loads. Steady flow simulations were conducted for Gemini, ${ }^{15}$ while more recent steady and unsteady CFD results are incorporated herein.

Wind loads are relevant both over the primary mirror $^{16}$ (M1) and on the secondary mirror (M2) and nearby supporting structure. Although the crosssectional area of M2 is small compared to that of M1, the structure around the mirror is exposed to higher wind speeds, and thus the resulting forces are also a significant contributor to image degradation, ${ }^{1}$ resulting in the motion of M2 and the deformation of M1 through structural coupling. Steady forces can be compensated by active control of the mirror surfaces. The optical consequences due to unsteady forces result primarily from the motion and deformation of M1 and M2 and can be computed from an integrated model that predicts the actively controlled structural response. ${ }^{2}$ The performance is less sensitive to the motions of M3 and Nasmyth platforms, and forces on these surfaces are not estimated. Wind loads on the dome could also be transmitted to the structure, although this effect is neglected herein. While air moving within the enclosure is bad for telescope vibration, some flow is necessary to ensure thermal equilibrium to avoid turbulence-induced dome seeing. ${ }^{17}$ Future telescope enclosures are likely to have vents that can be opened to increase air flow across the primary mirror, particularly in low external wind conditions.

The wind flow inside the dome involves several mechanisms. In addition to the mean flow pattern, there is broadband turbulence generated by the flow passing over and through the dome opening and also generated by the flow entering the dome through vents. For smooth, air-tight domes, it is possible to have significant tones associated with shear layer or Rossiter modes. ${ }^{18}$ These modes are attenuated by open venting and external structure and are not expected to lead to significant forces; the design issues required to ensure that this is true are described in Appendix A.

The relevant characteristics of the turbulence include the spatial distribution within the enclosure, the temporal spectrum, and the spatial correlation. None of the data sources described earlier gives a complete understanding of the wind loads. Furthermore, for design purposes, it is necessary to understand how wind loads vary with external wind speed and design parameters such as secondary mirror location and area or enclosure and aperture size, rather than simply applying a specified load pattern obtained (e.g., from CFD or experimentally) for a particular design and wind condition. The combination of existing data with additional CFD analyses is used herein to synthesize the required understanding and estimate relevant wind characteristics.
The next section lists key assumptions. Section 3 gives a brief overview of the different sources of data used to develop the parameterization. Section 4 provides details on both the physics and modeling of the broadband turbulent forces on the telescope.

\section{Assumptions}

The major assumptions made herein are listed below:

- Flow patterns and approximate velocity and pressure distributions are assumed to be robust to design details and can therefore be estimated from disparate data sources; this assumption is essential to this undertaking and is validated by the CFD (Fig. 6). The enclosure is assumed to be roughly hemispherical, e.g., a calotte or dome-shutter design. The most critical scaling parameter is the ratio of aperture to dome diameter, and the data used herein fall in a range of $28 \%-40 \%$. Clearly, the further that a dome design departs from the designs considered herein, the greater the error may be.

- The flow is turbulent (true for any large telescope) and the aperture sufficiently high above the ground to not have significant velocity gradients over the opening ( $\sim 20 \mathrm{~m}$ typically sufficient on mountain sites).

- Wind loads on the dome, tertiary mirror, and instrument platforms are ignored.

- The secondary mirror is assumed to be supported by legs similar to radio telescopes. The spider support typical for existing optical telescopes would significantly increase the cross-sectional area exposed to the wind for the upper part of the telescope.

- The forces on the secondary support structure are represented by an equivalent force on M2. This is a reasonable assumption at frequencies below the first resonance of the support structure, which is above the frequency range of interest. Matching the torque about the elevation axis is appropriate assuming that the dominant effect of forces on M2 is telescope rotation.

- The wind force on the secondary mirror and support structure are estimated from velocity measurements. This requires an assumption on the drag coefficient, and an assumption that the presence of the structure does not significantly alter the flow patterns in the dome. (This would clearly be a poor assumption for accurately estimating wind loads on the primary mirror.)

- A von Karman broadband turbulence spectrum is assumed, which is consistent with the data. The spatial correlation characteristics are assumed to be consistent with frozen turbulence.

- Turbulence is generated by flow through the aperture and through vents, but the effect of smaller structures (e.g., tertiary tower, baffles, and the telescope structure) is not included. Forces on small length scales are therefore not well represented.

- All forces are assumed to scale with the external dynamic pressure, $q=1 / 2 \rho U_{\infty}{ }^{2}$. For reference, the 50th and 90th percentile external wind speed $U_{\infty}$ measured by the Canada-France-Hawaii Telescope 
(a)

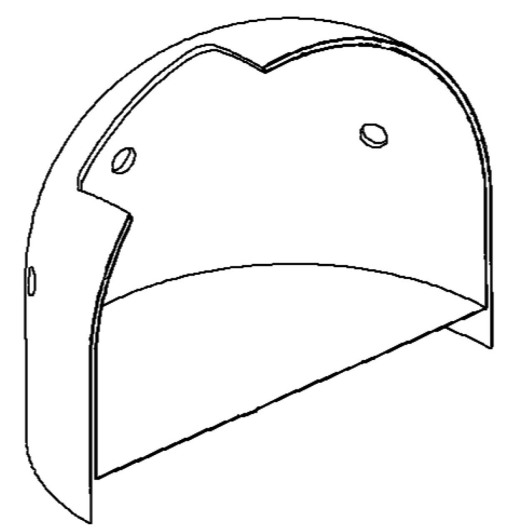

(b)

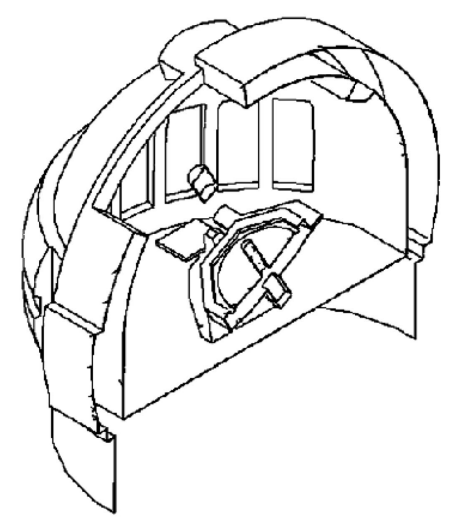

(c)

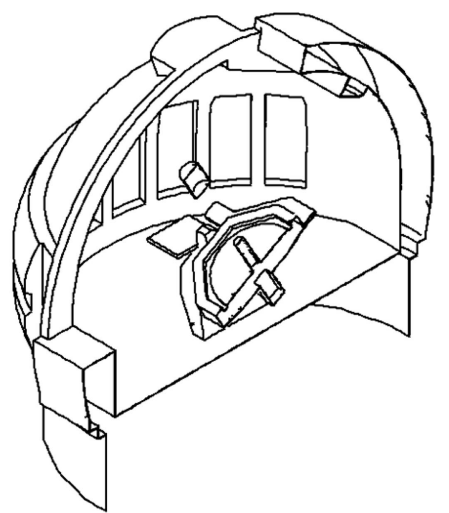

Fig. 1. Illustration of enclosure designs. (a) Simplified enclosure used in the M2 flow-field wind-tunnel test. Realistic (nonsmooth) enclosure designs based on the Gemini dome geometry were simulated with the CFD with (b) the shutter raised and (c) lowered to its minimum position.

(CFHT) on Mauna Kea are 6.5 and $13.5 \mathrm{~m} / \mathrm{s}$, respectively.

\section{Sources of Data}

Our understanding of the wind inside a telescope enclosure is informed by measurements at Gemini South, ${ }^{3,4}$ wind-tunnel experiments focused on the flow near M2 (Ref. 6), the loads on M1, ${ }^{13}$ and CFD. ${ }^{7}$ The wind-tunnel tests were conducted with simplified dome geometries that result in flow fields that are not identical to those expected in realistic domes, and CFD is used to understand the differences. The forces on M1 and M2 are estimated from Gemini data and M2 wind-tunnel data, while the other sources of information were used to aid understanding.

\section{A. Gemini}

Comprehensive wind data were collected during integration of the $8 \mathrm{~m}$ Gemini Southern Observatory. Reference 3 gives a full description of the experiment, and earlier analyses of the results appear in Refs. 4, 5 , and 19 . Velocity measurements were made using ultrasonic anemometers above the dome, behind the M2 assembly, and at three points around M1. The surface of the (dummy) primary mirror was instrumented with 32 pressure sensors. The sensors were recorded at $10 \mathrm{~Hz}$ for $300 \mathrm{~s}$, for 40 separate tests varying the azimuth angle with respect to the wind, telescope elevation angle, and the position of upwind and downwind vent gates. The lower shutter was not raised, and most of the data were collected with vents open. This configuration is appropriate for low external wind speeds to minimize thermal problems but is not optimal for higher external wind speeds where wind buffering becomes an issue. Therefore the Gemini data are not representative of the minimum wind loads that are achievable. The external wind is relatively constant in both speed $(\sim 10 \%$ standard deviation) and direction $\left(\sim 9^{\circ}\right.$ standard deviation) over the 5 min test intervals, allowing the internal wind speeds to be meaningfully defined as a ratio of the external wind speed for a given orientation.

\section{B. Wind Tunnel Tests}

The M2 flow-field wind-tunnel test ${ }^{6}$ collected data within a scaled telescope enclosure to understand the flow field around the region near the dome opening where M2 and its supporting structure would be subjected to wind loads. The model was representative of a generic empty telescope dome at approximately $1 \%$ of the expected full-scale enclosure size for a $30 \mathrm{~m}$ diameter telescope. The enclosure, shown in Fig. 1(a), is a hemisphere with diameter $D=0.83 \mathrm{~m}$ placed on a cylinder of height $H=0.28 \mathrm{~m}$. The interior floor is $H_{f}=0.09 \mathrm{~m}$ above the base. The $L=0.32 \mathrm{~m}$ square dome opening was fixed at $30^{\circ}$ from the zenith. A vented cross-sectional area up to $25 \%$ of the dome opening area could be achieved using 12 vents with a diameter of $D_{v}=5 \mathrm{~cm}$; data were collected with all of these closed, half open, and all open. The configuration corresponds to a full-scale enclosure with $D=90 \mathrm{~m}, H=30 \mathrm{~m}, H_{f}=10 \mathrm{~m}, L=35 \mathrm{~m}$, and $D_{v}=5.5 \mathrm{~m}$, reduced by a scale factor of $\sim 108$. The full-scale Reynolds number of the flow is sufficiently high $(\sim 20 \mathrm{M})$ so that the flow throughout the enclosure is turbulent. Provided that the experimental Reynolds number is sufficiently high to ensure that the flow is also turbulent, the flow is largely independent of the Reynolds number and therefore scale. The wind-tunnel tests were conducted at $35 \mathrm{~m} / \mathrm{s}$, higher than expected wind speeds, but yielding a sufficient $\operatorname{Re} \sim 2 \mathrm{M}$.

DPIV data were collected in a vertical plane near the dome opening to obtain the mean and fluctuation velocity. Data were collected only for azimuth angles of $0^{\circ}$ and $180^{\circ}$ with respect to the wind so that the mean flow along the centerline remained planar due to symmetry. Since the DPIV data cannot be recorded at a sufficient rate to give temporal spectra, hot-wire anemometry data was also collected along the telescope axis. The most significant error source in the DPIV data is the difference between the measured particle velocities and the actual flow velocities in regions of high flow acceleration where the particles may not accurately follow the flow. ${ }^{6}$ Significant errors 
are possible near the dome opening, and thus the predicted loads on M2 are computed from the error bound rather than the measured velocity.

\section{Computational Fluid Dynamics}

Three-dimensional steady and unsteady CFD simulations were conducted for a variety of telescope enclosure configurations, including wind-tunnel-scale [Fig. 1(a)] and Gemini enclosures [Figs. 1(b) and 1(c)] that allow validation of the computational results. The more realistic enclosure based on the Gemini geometry included vent gates, internal supports, faceted sides, girders, cranes, and other discontinuities. With the exception of wind-tunnel validation, simulations included a simplified telescope that omitted features less than $0.5 \mathrm{~m}$ in size. The truss structure under the primary mirror cannot be resolved and was replaced by a solid surface or omitted, and the structure of the secondary support was simplified or omitted.

The effects of turbulence were incorporated through modeling, and commercial software (STAR-CD) was used to solve the incompressible isothermal NavierStokes equations on a finite volume grid. Two types of turbulent modeling were used. The renormalization group (RNG) version ${ }^{20}$ of the $k-\varepsilon$ approach ${ }^{21}$ solves the Reynolds averaged momentum equations plus two additional equations for the turbulent kinetic energy and its rate of dissipation. Since $k-\varepsilon$ methods are dissipative, a large eddy simulation (LES) Smagorinski model ${ }^{22}$ was used to improve estimates of broadband turbulence; this approach explicitly solves for the large-scale turbulence motion, while subgrid-scale motions are approximated by a model. In addition to this modeling, the inlet turbulence is introduced as a zero-mean perturbation with a standard deviation of $\sqrt{2 / 3 k}$.

The geometry used in the wind-tunnel experiment was simulated to assess the ability of the CFD to capture turbulence for this problem. The computational domain was that of the M2 flow-field windtunnel test, with an artificially extended downwind section to facilitate the application of boundary conditions. The basic DPIV and optics equipment present in the measurements were incorporated. The spatial resolution of the computational grid varied from $0.01 \mathrm{~m}$ around and inside the model to $0.002 \mathrm{~m}$ normal to the surface of the model, while the temporal resolution was set at $0.2 \mathrm{~ms}$. The surface of the tunnel and model was given a smooth, no-slip boundary. The inlet air velocity was taken to be uniform with a speed of $35 \mathrm{~m} / \mathrm{s}$ and very low turbulent intensity. The outflow boundary condition set all normal derivatives to zero (convective exit).

For full-scale simulations, the enclosure was placed on a flat square plane nine dome diameters on each side, with the ceiling of the computational volume equal to half the domain length. The outflow boundary condition was convective, while the sides and ceiling of the computational domain were given free-slip boundaries with zero normal velocity. The surface of the enclosure and telescope structure was given a smooth,
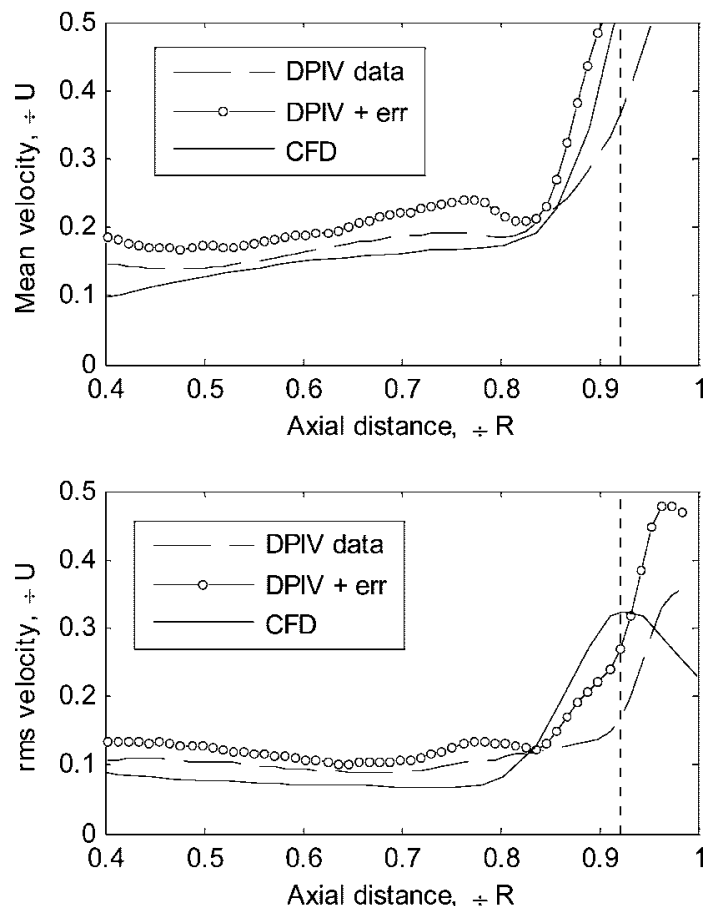

Fig. 2. Comparison between wind-tunnel DPIV data and the CFD. Data are on axis, position normalized by dome radius and the mean (upper) and unsteady rms (lower) normalized by external wind speed. For DPIV data, the actual particle velocity (dashed curve) and worst-case estimate of flow velocity (open circles) are shown.

no-slip boundary. The spatial resolution varied from several meters in the outer region of the volume, to $1 \mathrm{~m}$ inside and around the enclosure, $0.5-0.25 \mathrm{~m}$ on the telescope surface and $0.2-0.05 \mathrm{~m}$ normal to the structure. The sampling rate was $20 \mathrm{~Hz}$. The fluid properties used corresponded to air at $4000 \mathrm{~m}$. The horizontal incoming air velocity profile $u(z)=$ $U(z / h)^{1 / 7} \mathrm{~m} / \mathrm{s}$ reproduces that of a typical flat plate turbulent boundary layer; the average speed at the opening level was approximately $10 \mathrm{~m} / \mathrm{s}$. The incoming air turbulent intensity was $2 \%$, and the rate of turbulent energy dissipation was calculated from the turbulent kinetic energy level by assuming an atmospheric eddy length scale of $5 \mathrm{~m}$ and Kolmogorov equilibrium between energy production and dissipation.

The spatial distribution along the telescope axis of mean and rms unsteady velocity measured in the wind tunnel is compared with the computed values in Fig. 2 for a case with vents closed. Both the measured and the maximum velocity from the DPIV error estimates are plotted. A comparison of the turbulence power spectrum with Gemini data is shown in Fig. 3. Since the turbulence amplitudes tend to be underpredicted by CFD, Gemini and wind-tunnel data are used to estimate the wind loads. However, based on these results, the CFD is believed to capture the physics sufficiently well to understand amplitude changes with design parameters.

The CFD has also been used to verify that the mean flow pattern near M2 is not significantly affected by 


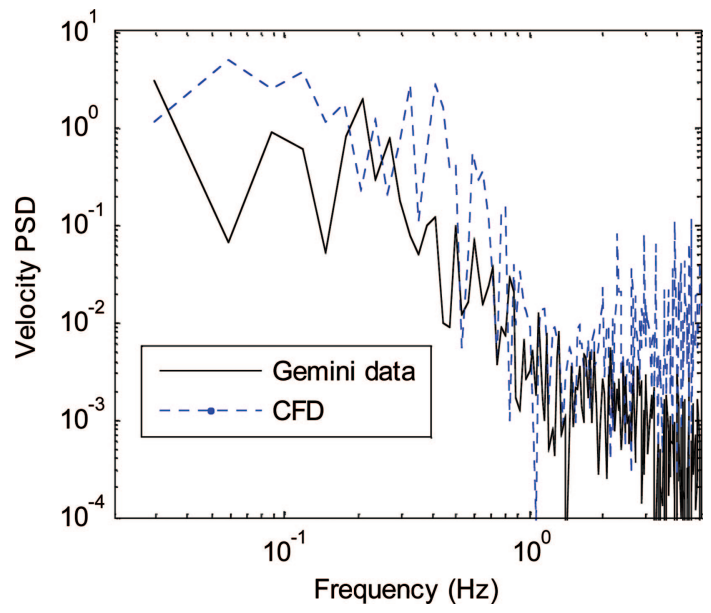

Fig. 3. (Color online) Comparison of the CFD velocity spectrum and spectrum from data measured at Gemini: $0^{\circ}$ azimuth, $30^{\circ}$ zenith angle, closed vents.

the presence of M2 and that there are no significantscale effects on wind loads (comparing wind-tunnel with full-scale simulations of identical geometry).

\section{Broadband Forces}

\section{A. Overview}

The mean flow pattern in the telescope enclosure is described in Ref. 6. For upwind orientations, some flow enters through the opening, the flow is down along the back of the enclosure, forward and slightly upward along the sides, and upward along the front. For downwind orientations, the opening is within the recirculation region of the enclosure wake, and thus the flow enters the enclosure through the dome opening in a direction opposite to that of the free stream.

Steady forces can be compensated by telescope control systems, and it is the unsteady forces that are of interest for predicting telescope image degradation. For notation, the velocity $u$ and pressure $p$ can be decomposed into their mean and fluctuating components as $u=\bar{u}+u^{\prime}, p=\bar{p}+p^{\prime}$, with

$$
\begin{aligned}
& p=1 / 2 \rho u^{2}=1 / 2 \rho\left(\bar{u}^{2}+2 \bar{u} u^{\prime}+u^{\prime 2}\right), \\
& \bar{p}=1 / 2 \rho \bar{u}^{2}+1 / 2 \rho \overline{u^{\prime 2}}, \\
& p^{\prime}=1 / 2 \rho\left(2 \bar{u} u^{\prime}+u^{\prime 2}-\overline{u^{\prime 2}}\right),
\end{aligned}
$$

where $\bar{u}$ is the mean velocity and $u_{\mathrm{rms}}=\left(\overline{u^{\prime 2}}\right)^{1 / 2}$ is the $\mathrm{rms}$ of the fluctuating (unsteady) velocity. The rms value of the fluctuating component of the dynamic pressure can be written in terms of an effective velocity:

$$
p_{\text {rms }}=\left(\overline{p^{\prime 2}}\right)^{1 / 2}=1 / 2 \rho u_{\text {eff }}^{2},
$$

where, for Gaussian velocity perturbations $u^{\prime}$, the definition above leads to

$$
u_{\mathrm{eff}}^{2}=\sqrt{\left(2 \bar{u} u_{\mathrm{rms}}\right)^{2}+2 u_{\mathrm{rms}}{ }^{4}} .
$$

The telescope dome significantly reduces the wind speeds inside the enclosure. The approach we take to estimating wind loads is to first estimate the effective velocity near M1 and M2 relative to the external mean wind speed $U_{\infty}$, and then to estimate the surface pressure and drag force from

$$
\begin{gathered}
p_{\mathrm{rms}}=C_{p}\left(1 / 2 \rho u_{\mathrm{eff}}^{2}\right), \\
F_{\mathrm{rms}}=C_{D} A\left(1 / 2 \rho u_{\mathrm{eff}}^{2}\right) .
\end{gathered}
$$

The expression in Eq. (7) for the force on M2 has recently been validated with simultaneous measurements of velocity and torque at Keck. ${ }^{10}$ The drag coefficient and area depend on particular telescope designs and are not discussed herein. Note that the pressure on a surface may be lower than the nearby dynamic pressure away from the surface; estimates for the local pressure coefficient $C_{p}$ are given in Fig. 8 . We first discuss the velocity estimates at M1 and M2, and then the temporal spectrum and spatial correlation in Subsection 4.D.

\section{B. Wind Speed near M2}

Measurements from the wind-tunnel test of the Keck enclosure concluded that the wind speeds near the secondary mirror would be roughly $25 \%$ of the external wind speed. ${ }^{11}$ For upwind orientations with the shutter down, the Gemini data indicate $40 \%$ of the external wind speed at M2 and less than 10\% at M1. Wind-tunnel DPIV data suggest that lower wind speeds are possible if the size of the dome opening is minimized; the CFD is used to verify this conclusion. Note that near M2, the flow velocity is nearly orthogonal to the telescope optical axis, and the velocity in the axial direction is much smaller than the transverse component. Developing estimates of the force on M2 and the supporting structure is also complicated by the desire to predict these forces as a function of the location of the mirror within the dome (i.e., to estimate the benefit from building a slightly larger dome with greater clearance between the secondary mirror and the shear layer) and also to estimate forces on the support structure.

The mean and perturbation rms velocity measured at Gemini near M2 are shown as a function of the external wind speed in Fig. 4. Each plotted data point corresponds to an upwind $\left(0^{\circ}\right)$ test condition and either a different zenith angle or a different vent configuration. Data sets for which any of the velocity sensors were saturated were not included. The mean wind for a $0^{\circ}$ orientation is on average $40 \%$ of the external wind speed, the rms perturbation (turbulence component) is on average $23 \%$ of the external wind speed, and the effective turbulent velocity [Eq. (5)] averages $43 \%$.

The wind speeds are much lower in the data from the wind-tunnel test, as shown in Fig. 5, from DPIV data. As noted in Appendix A (see Fig. 13 and also Ref. 6), the wind-tunnel DPIV data near the dome opening with vents open are dominated by the broad- 


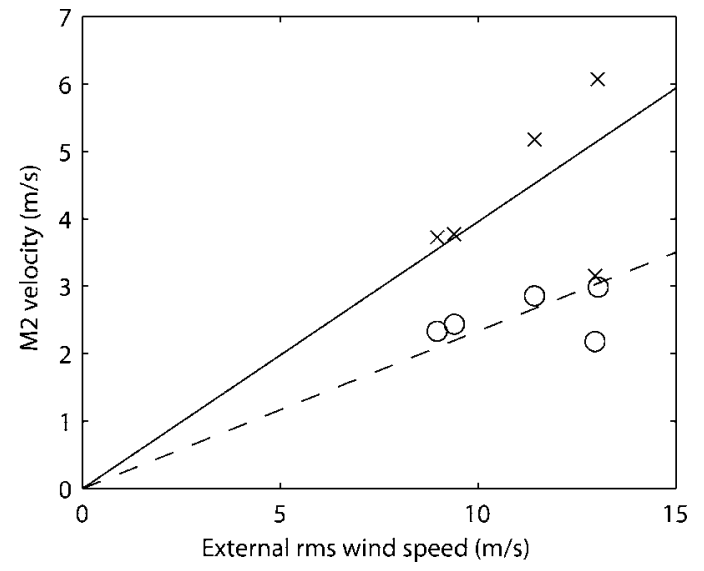

Fig. 4. Mean (solid line, $\times$ ) and rms fluctuation (dashed line, $\bigcirc$ ) velocity near M2 measured at Gemini for upwind orientations, as a function of external rms wind speed.

band turbulence, rather than the shear layer mode. The DPIV data without vents open contain significant energy associated with the shear layer mode, rather than solely broadband turbulence, and are not considered herein. The normalized effective turbulent velocity $u_{\text {eff }} / U_{\infty}$ along the telescope optical axis is plotted for both upwind ( $0^{\circ}$ azimuth) and downwind $\left(180^{\circ}\right.$ azimuth) in Fig. 5. The plot is of the upper bound on velocity from the DPIV error analysis. For small variations in the size of the dome compared to the dome opening it would be reasonable to normalize the data by the critical radius $R_{c}{ }^{2}=(D / 2)^{2}-$ $(L / 2)^{2}$ at which the line connecting the edges of the aperture intersects the telescope axis, rather than by the dome radius; this radius is indicated by the vertical dashed lines. Padin and Davison ${ }^{1}$ have suggested that the internal velocities should also scale with $(D / L)^{2 / 3}$. For the wind-tunnel model, $L / D=0.38$.

Both the mean and the effective velocity at M2 are between 0.15 and 0.2 of the external mean wind

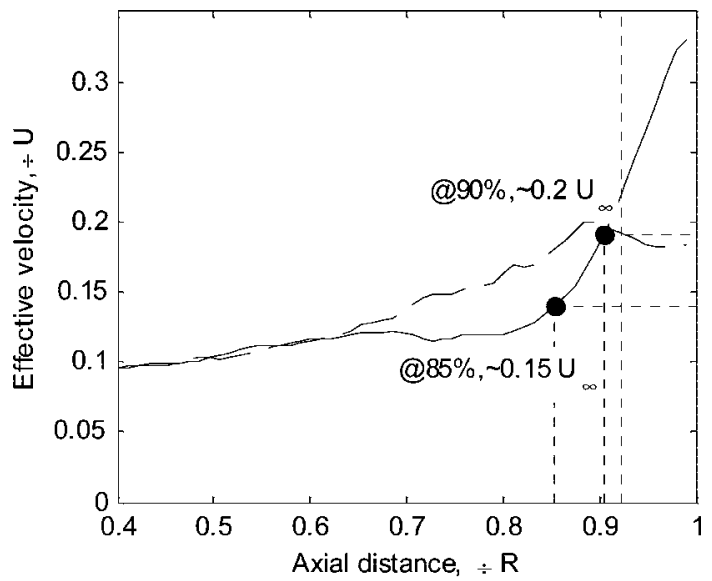

Fig. 5. Effective transverse broadband turbulent velocity near M2 and supporting structure, from wind-tunnel DPIV data as a function of axial distance from the center of the dome, upstream orientation (solid curve) and downstream (dashed curve). The vertical line is at $R_{c}$.

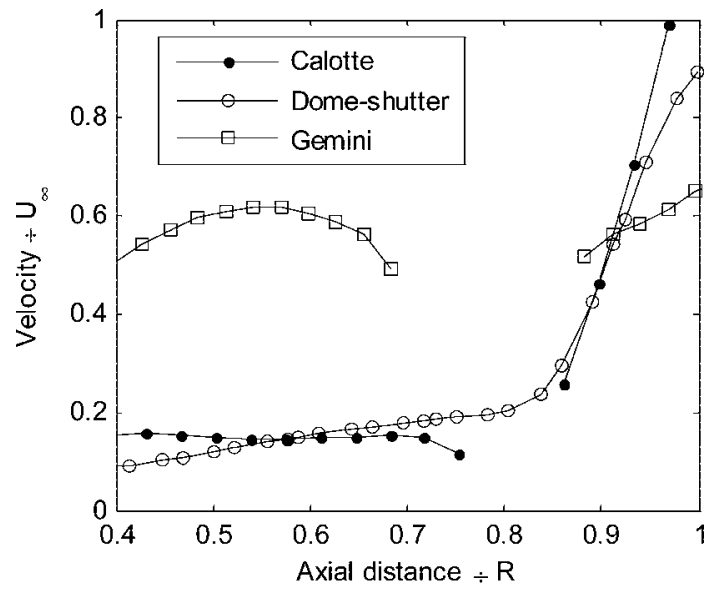

Fig. 6. Comparison of computed upwind axial velocity profiles for different domes. The calotte $(\bullet)$ and Gemini $(\square)$ cases include a telescope structure, and the gap in the plot corresponds to the location of M2.

speed, depending on the location of $\mathrm{M} 2$ relative to the shear layer. There is a significant drop in wind speed as one moves through the shear layer, while the velocity over most of the M2 support structure is relatively constant. Note that analysis of the Keck wind-tunnel data hypothesized a linear decrease in wind speed with radial location; this hypothesis was based on extrapolation from limited data taken only for large fractions of the dome radius.

One difference between data sources is the uniform external wind speed in the wind-tunnel test, while the real environment involves some low-frequency variation in wind speed and direction. However, while this may be a factor (see Fig. 9), it does not explain a significant fraction of the discrepancy.

The Gemini data were collected with the shutter lowered, so that the open area is much larger than in the wind-tunnel model, and the shear layer will extend deeper inside the enclosure. CFD analyses (Fig. 6) of the wind-tunnel and Gemini configurations agree qualitatively with the experimental data, confirming that the open area is the reason for the higher wind speeds observed at Gemini. It is therefore reasonable to expect that wind loads closer to those estimated from wind-tunnel data are more representative provided that the shutter can be closed. Figure 6 also illustrates that while the internal flow speeds are sensitive to the size of the dome opening, they are relatively insensitive to details of the design. Included in the plot are CFD results for the domeshutter (square opening, comparable to wind-tunnel) and calotte (round opening) designs. When estimating wind speeds near M2 to be $20 \%-25 \%$ of external, the two designs are equivalent. What matters, from the perspective of wind loads on $\mathrm{M} 2$, is the location of the shear layer that develops across the opening relative to the location of M2.

While the wind-tunnel data were only collected for $0^{\circ}$ and $180^{\circ}$ azimuth angles with respect to the wind, the Gemini data were collected for a variety of azimuth and zenith angles. However, when the dome opening 
was facing downwind, the anemometer mounted above the dome was in disturbed air flow, giving a less accurate estimate of the external wind speed. Because of this, and the simultaneous variation in orientation and venting configuration, it is difficult to reliably estimate the effect of orientation beyond noting that upwind is the worst case. In the M2 wind-tunnel data, the turbulence is not significantly lower for downwind observations; at some locations, the downwind orientation has higher effective turbulence due to the entrainment of the wake flow. The minimum open aperture area significantly reduces the wind loads for upwind orientations and should be expected to lead to a smaller variation in wind loads between upwind and downwind orientation.

\section{Wind Speed near M1}

Estimates of the pressure on the surface of the primary mirror can be derived from the differential pressure measurements collected at the Gemini observatory. Converting these into estimates of the distributed force requires consideration of the spatial correlation described in Subsection 4.D.

Note that pressure variations on a surface can be separated into those generated by local turbulence passing over the surface, with a (short) correlation length related to frequency and the local wind speed, and acoustic perturbations generated by turbulence elsewhere, with a (long) correlation length related to frequency and the speed of sound. For turbulenceinduced pressure variations, the correlation between the front and back of the primary mirror is small. However over the frequency range of interest, acoustic pressure variations have wavelengths many times the dome diameter and produce essentially zero net force on the mirror. Absolute pressure measurements are sensitive to both components. The wind-tunnel measurements in Ref. 13 are dominated by the acoustic component and thus cannot be used to estimate wind forces.

Measurements taken at the Gemini observatory with the vents closed and the telescope facing upwind showed, on average, that the effective velocity $u_{\mathrm{M} 1, \text { eff }}$ near M1 was $7 \%$ of the external wind speed ${ }^{19}$ (Fig. 7), and that this was a good predictor of both the rms pressure $\left(C_{p} \sim 1\right)$ on the primary mirror and the outer-scale frequency. The mean velocity for these cases is roughly $3 \%$. Measurements taken with the vents open showed, on average, that the mean velocity near M1 was $30 \%$ of the external wind speed ${ }^{5}$ and the effective velocity was $\sim 35 \%$; these are averages over different orientations and different vent configurations. The local pressure coefficient relating unsteady surface pressure to local dynamic pressure for the vented data is $\sim 0.25$ as shown in Fig. 8. The Gemini data were collected with a flat dummy mirror in place of M1, which was slightly recessed. The $0.4 \mathrm{~m}$ higher cylinder that surrounded the dummy mirror may have resulted in slightly reduced pressure fluctuations than would be representative of the actual mirror, and thus the actual pressure coefficient may be higher.

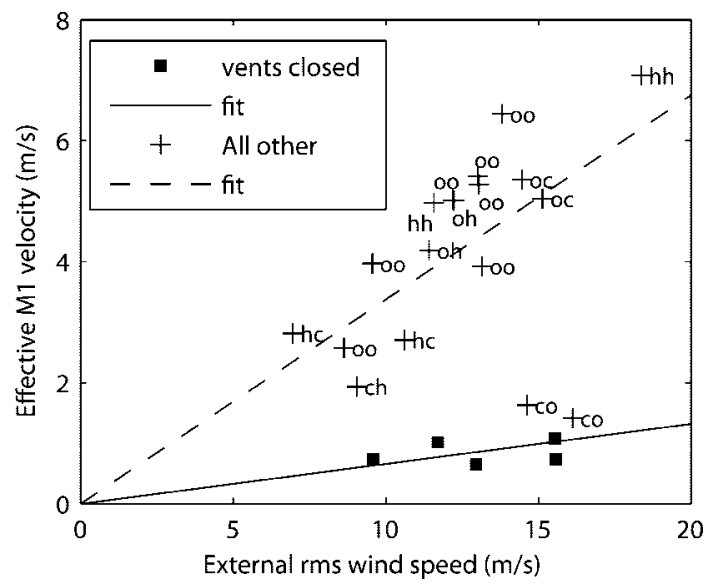

Fig. 7. Effective velocity near M1 from Gemini data as a function of external wind speed. Solid points and line correspond to cases with vent gates closed. The vent configuration for other cases is labeled o for open, $\mathrm{h}$ for half, c for closed; the first letter is the upwind vent, and the second is the downwind vent.

\section{Temporal Spectrum and Spatial Correlation}

While the turbulence inside the telescope enclosure is clearly not isotropic, observations at Gemini 5,19 (e.g., Figs. 9 and 10) and other telescopes (Keck, VLT, MMT) as well as measurements in wind-tunnel testing (see Fig. 12) and computational results suggest that the broadband turbulence spectrum inside the enclosure can be reasonably approximated with Kolmogorov scaling, giving a velocity power spectrum with $f^{-5 / 3}$ frequency dependence, while the spectrum of the pressure at a single point has a $-7 / 3$ slope.

Incorporating the outer scale with a von Karman spectrum matches the data; the pressure spectrum is thus proportional to

$$
\Phi_{\mathrm{VK}}(f)=\left[\frac{2 \sqrt{\pi}}{\Gamma(2 / 3) \Gamma(5 / 6)}\right] \frac{1 / f_{0}}{\left[1+\left(f / f_{0}\right)^{2}\right]^{7 / 6}} .
$$

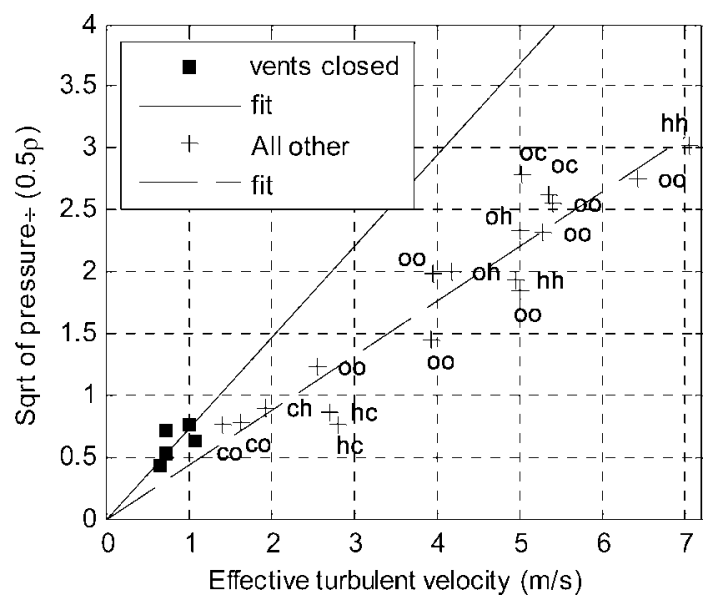

Fig. 8. Comparison between unsteady pressure on M1 and effective velocity from Gemini data as a function of external wind speed for estimating local pressure coefficient. Solid points and line correspond to cases with vent gates closed. Vent labeling is as in Fig. 7. 


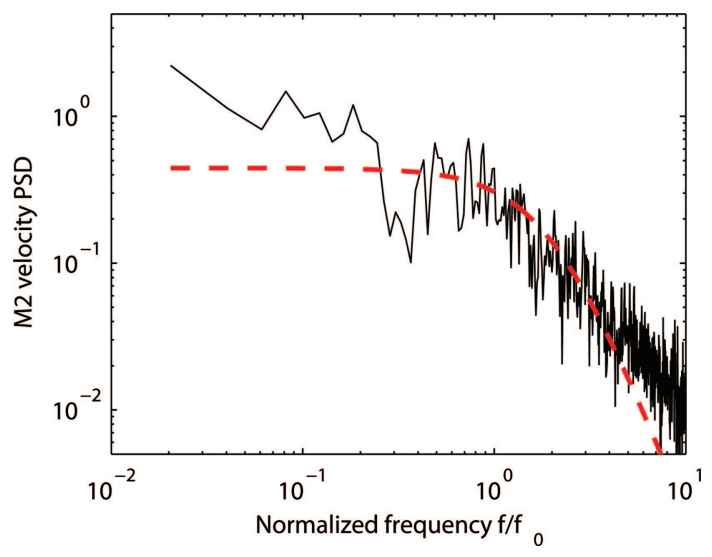

Fig. 9. (Color online) M2 velocity spectrum for one case, vents closed, compared with a von Karman spectrum. The rms velocity is $13 \%$ higher than the rms of the fit due to the higher amplitudes at very low frequencies.

The frequency $f_{0}$ is related to the outer scale of turbulence by the local convective velocity. The outer scale associated with the turbulence observed near M2 and the turbulence near M1 when the vents are closed is roughly equal to the diameter $L$ of the dome opening ${ }^{5,19}$ for upwind orientations, implying that the physical mechanism that creates the turbulence is due to the flow passing over and through the open aperture, rather than the ingestion of free-stream turbulence from the outside air. The latter effect is small in the data available from Gemini, although variations in the external wind may result in additional energy at very low frequencies, as shown in Fig. 9. This plot is not representative; typical variations in external wind speed are at much lower frequency and of much lower energy. For downwind orientations, significant turbulence is generated by the wake of the dome, and the relevant outer scale is the dome diameter, rather than the diameter of the opening. The outer scale associated with the turbulence over M1 that is caused by flow through open vents is related to the height of the vent gates. ${ }^{5}$ (See, e.g., the spatially averaged M1 pressure spectrum from the Gemini data in Fig. 10, with and without open vents, both at $0^{\circ}$ azimuth).

All of the measured data available lead to estimates of pressure, but it is the force obtained by integrating pressure over a finite area that is of relevance to predicting telescope performance. This spatial integration introduces an additional spectral factor,

$$
\Phi_{S}(f)=\left[1+\left(f / f_{s}\right)^{2}\right]^{-1},
$$

multiplying the von Karman spectrum to give a total $-13 / 3$ slope, with the corner frequency $f_{s}$ from this additional factor depending on the wind speed and the spatial scale of the integration. The spatial integration accounts for the frequency dependence of the spatial correlation length assuming frozen turbulence. This effect was incorporated in some prior mod-
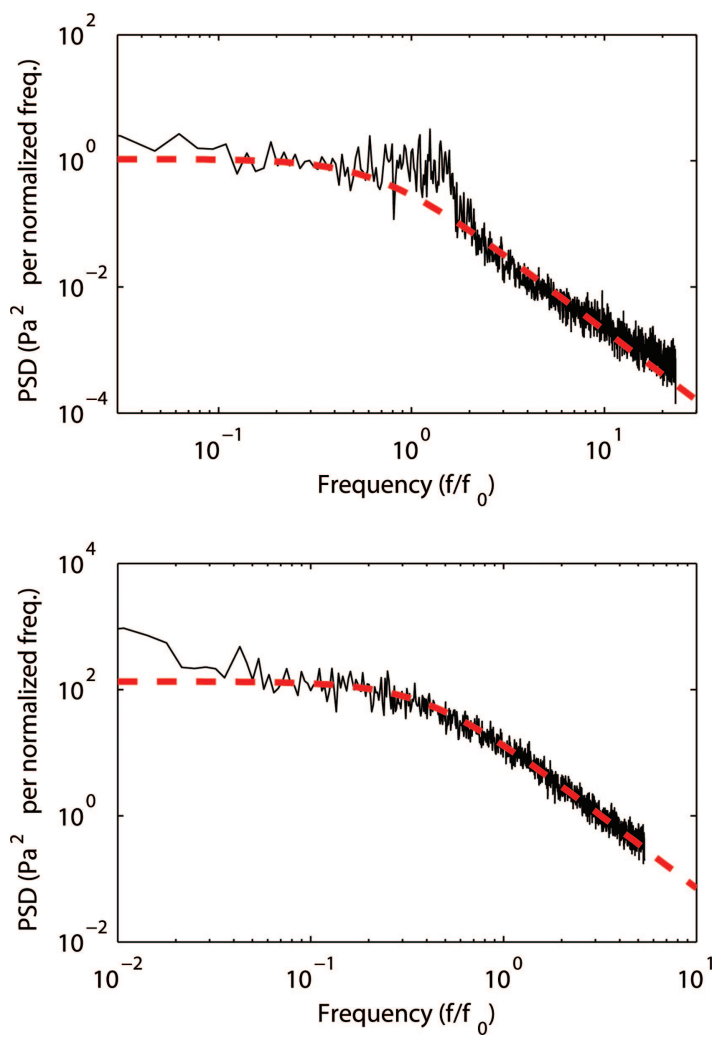

Fig. 10. (Color online) Representative M1 pressure spectra measured at Gemini, compared with von Karman spectrum (dashed curve); without venting (upper), and with open vents (lower).

els with an empirically determined aerodynamic admittance factor that multiplied the velocity power spectrum by a factor with frequency dependence $f^{-8 / 3}$ to give the correct observed behavior. ${ }^{23-25}$ The $-13 / 3$ slope can also be obtained from Kolmogorov scaling arguments.

First, consider the net force on a thin structure aligned with the mean wind velocity $U$. Frozen turbulence implies that the pressure on the surface at frequency $f$ corresponds to a particular spatial wavenumber $k=2 \pi f / U$, so that at any instant in time

$$
p(x)=\sqrt{2} p(f) \operatorname{Re}(\exp \{i[k x+\varphi(t)]\}),
$$

where $p(f)$ is the rms pressure at frequency $f$, obtained from the PSD and $\operatorname{Re}(\cdot)$ denotes the real part. The total force over a structure of length $L$ and width $w$ is given by

$$
\begin{aligned}
F & =w \int_{-L / 2}^{L / 2} p(x) \mathrm{d} x \\
& =\sqrt{2} p(f) \operatorname{Re}[\exp (i \varphi)] w L \operatorname{sinc}(k L / 2) .
\end{aligned}
$$

The rms force is obtained by averaging over the timedependent phase $\varphi$; this yields a factor of $1 / \sqrt{2}$. The maxima of the sinc function can be approximated by a first-order low-pass filter with unity gain at zero 
frequency and corner frequency $k L / 2=1$ or $f=$ $U /(\pi L)$. The overall temporal force spectrum that results is thus the product of the original von Karman pressure spectrum with the corner frequency set by the turbulence outer scale, and an additional attenuation factor with a corner frequency that is a function of the spatial length scale of the structure. Both factors involve the same convective velocity to scale between spatial and temporal frequencies. Therefore if one is considering structures that are small compared to the outer scale of turbulence (e.g., M2 itself), one can ignore the structural spectral factor. The integration, however, is important for understanding the loads on the M2 support structure and the distributed loads on M1.

The equivalent load $F_{\text {eq }}$ on M2 due to the loads on the supporting structure, chosen to match the torque about the elevation axis, is given by the integral

$$
L F_{\text {eq }}=w \int_{0}^{L} x p(x) \mathrm{d} x .
$$

The relevant velocity scaling between spatial and temporal frequencies is the projection of the assumed mean velocity along the support structure. Note that the wind-tunnel test data (e.g., Fig. 5) show that the unsteady velocities and therefore the unsteady pressure are roughly independent of location within the enclosure for radii below the shear layer. While the secondary mirror may be in a region of higher velocity close to the shear layer, most of the support structure will be in the more quiescent interior of the dome.

Integrating and computing the mean-square value over the phase $\varphi$ of the pressure gives the equivalent mean-square force as

$$
\begin{aligned}
F_{\text {eq }}(f)^{2}= & 2 L^{2}[1-\cos (k L)-k L \sin (k L) \\
& \left.+(k L)^{2} / 2\right] /(L k)^{4} p(f)^{2} w^{2} .
\end{aligned}
$$

This can be reasonably approximated, as shown in Fig. 11, by

$$
F_{\text {eq }}(f)^{2}=\left(L^{2} / 4\right)\left[1+(k L / 2)^{2}\right]^{-1} p(f)^{2} w^{2} .
$$

The appropriate spectrum of force on M2 to simulate this torque is therefore the force $F_{\text {leg }}(f)^{2}=$ $\left(w^{2} L^{2} / 4\right) \Phi(f)$ that would be correct at zero frequency and includes the spectral dependence $\Phi(f)$ of the pressure, multiplied by the additional roll-off that accounts for the correlation effects upon integration, with corner frequency $f=U /(\pi L)$.

Noting that the pressure at the top of the support structure is correlated with the force $F_{\mathrm{M} 2}$ on M2 itself, the approach can be extended to show that the net mean-square force has a spectrum that can be approximated by

$$
\begin{aligned}
F_{\mathrm{M} 2, \mathrm{net}}(f)^{2}= & F_{\mathrm{M} 2}(f)^{2}+\left[2 F_{\mathrm{M} 2}(f) F_{\text {leg }}(f)+F_{\text {leg }}(f)^{2}\right] \\
& \times\left[1+(k L / 2)^{2}\right]^{-1} .
\end{aligned}
$$
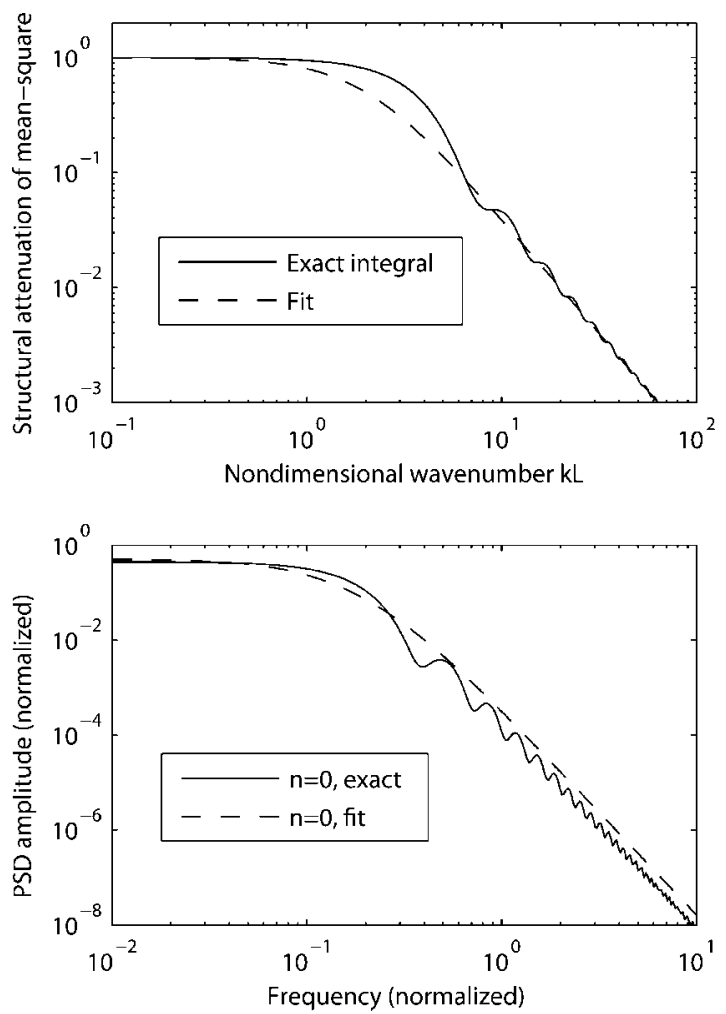

Fig. 11. Exact calculation of frozen-turbulence wind spectrum compared with a simple fit. For one-dimensional integration (upper), the structural attenuation is shown. For two-dimensional integration, the full spectrum is shown for the $n=0$ Zernike basis function; the final slope is $-13 / 3$.

Appropriate forces on M1 are more difficult to estimate not just because the structure is two-dimensional, but because we are interested in more than just the total integrated force. Rather than defining a frequencydependent spatial correlation matrix for loads on M1, a computationally simpler approach from Padin ${ }^{16}$ is to compute the spectrum corresponding to each term in a Zernike expansion of the force distribution on M1. This converges because for the turbulence caused by the flow through the dome opening or vent openings, the wind energy decreases rapidly for shorter length scales. Additional short-length-scale turbulence resulting from the flow across telescope structural elements is not explicitly considered herein but could be bounded from the maximum pressure observed and added to the long-length-scale turbulence represented by the Zernike expansion. It is possible to construct a basis so that the correlation between forces along different basis functions is minimized. However, the neglected correlation between forces applied to different Zernike basis functions $Z_{\mathrm{nm}}$ is small, and therefore the use of more familiar basis functions is sufficient.

The approach follows Padin ${ }^{16}$ with minor modifications. Each temporal frequency is associated with a given spatial frequency in the wind direction, which we arbitrarily choose as aligned with the $x$ axis. Integration of the sinusoidal pressure projected onto a basis function can be computed with the Fourier transform of the windowed basis function, with $W(r)=1 / \pi$ for 
the normalized radius $r \leq 1, W(r)=0$ otherwise, chosen to give results normalized by the total force $A p_{\text {rms }}$. The Fourier transform of $W(r) Z_{\mathrm{nm}}(r)$ is

$$
Q_{\mathrm{nm}}(\mathbf{k})=(-1)^{(n-m) / 2} \sqrt{n+1} J_{n+1}(2 \pi \mathbf{k}) / \pi \mathbf{k},
$$

with an additional factor of $\sqrt{2} \sin (m \varphi)$ or $\sqrt{2} \cos (m \varphi)$, whose average over all possible wind directions over M1 is unity. The complex wavenumber is $\mathbf{k}=$ $k \exp (i \varphi)=\left(k_{x}{ }^{2}+k_{y}{ }^{2}\right)^{1 / 2} \exp (i \varphi)$.

The nondimensionalized wavenumber in the wind direction is $k_{x}=f R / U$ (defined without the factor of $2 \pi$ for simplicity). The spatial frequency $k_{y}$ in the orthogonal direction, however, can take any value and thus the total mean-square force at frequency $f$ projected onto a Zernike basis function of radial degree $n$ is obtained by integrating over wavenumber $k_{y}$ :

$$
\begin{aligned}
F_{\mathrm{nm}}^{2}(f R / U)= & \frac{2 \sqrt{\pi}}{\Gamma(2 / 3) \Gamma(5 / 6)} \frac{n+1}{\pi^{2}} \\
& \times \int_{-\infty}^{\infty} \frac{\left[J_{n+1}(2 \pi k)\right]^{2}}{k^{2} k_{0}\left[1+\left(k / k_{0}\right)^{2}\right]^{7 / 6}} \mathrm{~d} k_{y}\left(A p_{\mathrm{rms}}\right)^{2} .
\end{aligned}
$$

The integrand is the amplitude of the pressure sinusoid at the frequency of interest and particular $k_{y}$ from the von Karman spectrum, multiplied by the Fourier transform of the windowed basis function. This integral can be computed numerically and approximated as before by the product of two termsthe original von Karman pressure spectrum and a second-order roll-off due to the integration over the structure with amplitude and corner frequency:

$$
\begin{aligned}
& A_{n} \approx(n+1)^{-2}, \\
& f_{n} \approx(U / 2 \pi R)(n+1)^{3 / 2} .
\end{aligned}
$$

The corner frequency increases with increasing radial degree while the zero-frequency amplitude decreases. This fit is shown with the full integral for $n=0$ in Fig. 11; the quality of the fit decreases with increasing $n$.

\section{Summary}

The ability to predict the performance of an observatory during its design phase is essential. One factor in this performance results from the wind-induced vibration of the telescope structure, caused by unsteady flow within the enclosure. Based on recently available data from full-scale measurements, scaled windtunnel tests, and new computational fluid dynamics analyses, a significant advance in our understanding of the wind loads is possible. The dependency of the wind loads on relevant telescope design parameters (M2 location, aperture size) and external wind speed has been described.
The predominant unsteady wind mechanism inside the telescope enclosure is broadband turbulence that can be approximated with a von Karman spectrum. The amplitude of the turbulence is high within the shear layer that exists over the opening of the dome, but the amplitude drops significantly a short distance below this shear layer. An additional source of turbulence is due to flow passing through open vents that may be used to control the thermal environment within the enclosure. A third source of unsteady loads results from tonal shear layer modes, which may couple with the Helmholtz mode of the cavity. While these tones are significant in some wind-tunnel and computational results, they are not believed to be a strong contributor to telescope vibration in more realistic geometries owing to a combination of effects. Venting decreases the coupling with the Helmholtz mode, while surface features on the dome exterior interfere with the shear layer vorticity development.

The amplitude of the equivalent force on M2 can be obtained from velocity estimates using an assumed drag coefficient [Eq. (7)]. For a dome with a minimum aperture, the velocity near M2 is less than $20 \%$ of external, depending on the location within the dome, while the velocity over the support structure is $\sim 0.1 U_{\infty}$. This is significantly lower than the wind speeds measured at Gemini without the lower shutter raised. Computational fluid dynamic analyses capture the difference in wind speeds with the dome open area. The equivalent force spectrum on M2 that captures the effect of the forces on the support structure has an additional roll-off relative to the pressure spectrum to account for the spatial decorrelation [Eq. (15)]. The amplitude of pressure forces on M1 with vents closed (best case) is roughly $p_{\text {rms }} \sim 1 / 2 \rho$ $\left(0.07 U_{\infty}\right)^{2}$; with vents open, much larger velocities are possible near M1. The spatial characteristics can be represented by a decomposition onto Zernike basis functions with amplitude and spectrum dependent on radial degree [Eqs. (18) and (19)].

\section{Appendix A: Shear Layer Mode}

The shear layer modes observed in wind-tunnel tests of simplified dome geometries, ${ }^{6,13} \mathrm{CFD}$ analyses of these same geometries, ${ }^{7,13}$ and also in airborne observatories ${ }^{26}$ result from flow passing over an open cavity. ${ }^{18}$ The differential flow speed across the shear layer generates vorticity, which rolls up into large vortices. As these encounter the end of the cavity opening, an acoustic reflection propagates upstream and interacts with the flow at the initial separation point. This feedback leads to an organization of the shear layer vorticity into modes with $n$ vortex structures across the length of the cavity opening. The characteristics of these modes include a large oscillatory vortex structure near the secondary mirror and supporting structure and a large oscillatory acoustic pressure throughout the entire enclosure. For downwind orientations, the aperture is within 


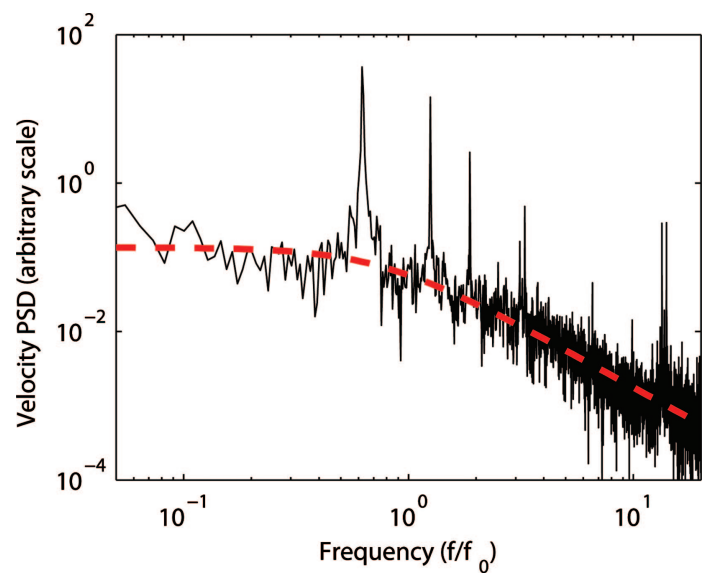

Fig. 12. (Color online) Velocity spectrum measured in a wind tunnel using a hot-wire anemometer for upwind orientation. The frequency scale is normalized by the convective time scale across the opening. The dashed curve is a fit with a von Karman spectrum.

the dome wake, and there can be no shear layer modes.

A typical spectrum including these modes is shown in Fig. 12. The frequency observed in wind-tunnel tests and CFD is roughly $f_{n}=0.66 n U_{\infty} / L$ for integer $n$. The factor of 0.66 corresponds to the shear layer convection speed across the opening; roughly the average of the speed on either side of the opening, with the local free-stream velocity at the leading edge approximately $10 \%$ higher than $U_{\infty}$ (from CFD). This is consistent with other estimates of the frequency for deep open cavities. ${ }^{27}$ The acoustic amplitude observed in wind-tunnel tests can be as high as $25 \%$ of the free-stream dynamic pressure $1 / 2 \rho U_{\infty}^{2}$. However, the shear layer mode strength is influenced by the damping of the Helmholtz resonance (see, e.g., Ref. 28 ), which is influenced by the vented area. ${ }^{6}$ The amplitude is also influenced by any external structure of the dome, which can affect the boundary layer thickness or the shear layer spanwise vorticity distribution (e.g., by introducing streamwise vorticity ${ }^{29}$ ). Finally, the net effect on the mirrors results from the differential pressure across them. Since the shear layer modes affect M1 through long wavelength acoustic pressure waves, the front and back of the primary mirror are exposed to similar pressure. Including all of these factors leads to the conclusion that while the shear layer modes are significant for certain orientations in wind-tunnel (e.g., Fig. 12) and CFD results, they are not the most important source of unsteady wind loads on the telescope structure.

The vented area required to obtain a significant reduction in the amplitude of the shear layer mode is relatively small (relative to the open area of the dome) as shown in Fig. 13 for hot-wire measurements of velocity near M2 in the wind-tunnel test. The total mean-square velocity in the first three shear layer modes was computed, normalized by the total meansquare velocity in the broadband turbulence. Note that the force is proportional to the square of the velocity, so a $25 \%$ vented area leads to a factor of 25

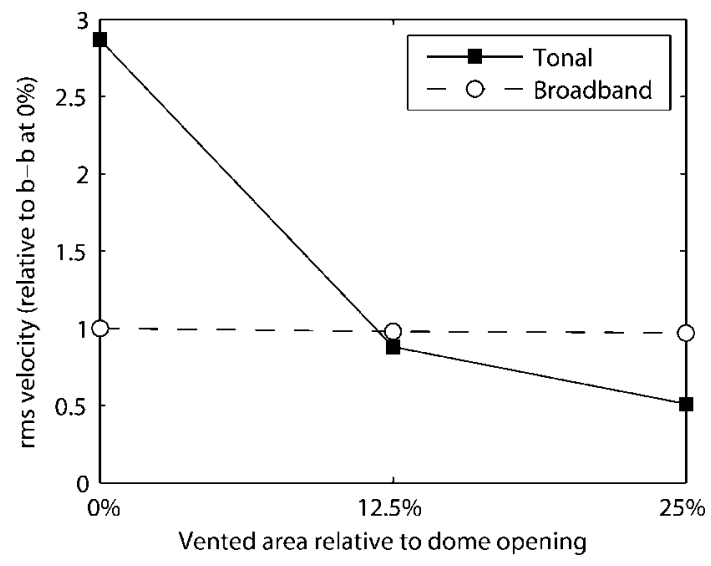

Fig. 13. Effect of venting on shear layer amplitude. The integrated mean-square velocity for the shear layer modes (solid line) is computed at one location near M2 from wind-tunnel measurements with three different levels of vented area. The residual broadband turbulence amplitude (dashed line) is nearly constant.

reduction in force. Note that, in contrast to the loads on M1, the broadband loads on M2 are not significantly influenced by the open venting. Based on the M1 wind-tunnel test, the reduction in the shear layer mode pressure on M1 with venting is similar to the reduction in the forces on $\mathrm{M} 2$, although the mechanism by which the shear layer mode affects M1 and M2 differs.

Estimates for the reduction in shear layer mode amplitude due to dome effects have been made from CFD simulations. The smooth dome case in Fig. 1(a) with no vents and the shutter closed to the minimum aperture was compared with simulations with a Gemini dome, both with the lower shutter raised, and in its lowest position [Figs. 1(b) and 1(c)]. With the shutter closed to its minimum aperture, the shear layer tone was reduced by approximately $50 \%$, due to the nonsmooth external dome surface. Lowering the shutter eliminated the tone completely. For this geometry, the incoming flow at the upstream edge of the opening has not yet been turned parallel to the surface, and there is no shear layer mode. Note that the typical observing condition at both the Gemini and Keck Observatories has the lower shutter completely open.

If shear layer modes are present, the resulting pressure field across M1 is uniform because of the long acoustic wavelength, this is confirmed by wind-tunnel data. (For example, a $0.25 \mathrm{~Hz}$ mode corresponding to a large telescope at high wind speeds would have a wavelength longer than $1 \mathrm{~km}$.) The forces on the secondary and supporting structure are nearly in phase with the forces on M1, but larger pressures are possible due to the drag caused by the large oscillatory velocities. The worst-case shear layer mode amplitude on M2 could be estimated from wind-tunnel measurements of an unvented enclosure.

The authors gratefully acknowledge the support of the Thirty Meter Telescope partner institutions. They are the Association of Canadian Universities for Re- 
search in Astronomy, the Association of Universities for Research in Astronomy (AURA), the California Institute of Technology, and the University of California. This work was supported, as well, by the Canada Foundation for Innovation; the Gordon and Betty Moore Foundation; the National Optical Astronomy Observatory, which is operated by AURA under a cooperative agreement with the National Science Foundation; the Ontario Ministry of Research and Innovation; and the National Research Council of Canada.

This work has benefited from the comments and constructive criticism of Terry Mast (University of California, Santa Cruz) and two anonymous reviewers. Various people were instrumental in collecting and interpreting the data used herein. In particular, Myung Cho conducted much of the early analysis of the Gemini data, and Tait Pottebaum conducted the M2 flow-field wind-tunnel test.

\section{References}

1. S. Padin and W. Davison, "Model of image degradation due to wind buffeting on an extremely large telescope," Appl. Opt. 43, 592-600 (2004).

2. D. G. MacMynowski, C. Blaurock, G. Z. Angeli, and K. Vogiatzis, "Modeling wind-buffeting of the Thirty Meter Telescope," in Modeling, Systems Engineering and Program Management for Astronomy II, M. Cullum and G. Angeli, eds., Proc. SPIE 6271, 62710M (2006).

3. M. K. Cho, L. Stepp, and S. Kim, "Wind buffeting effects on the Gemini 8 m primary mirrors," in Optomechanical Design and Engineering, A. Hatheway, ed., Proc. SPIE 4444, 302-314 (2001).

4. M. K. Cho, L. M. Stepp, G. Z. Angeli, and D. R. Smith, "Wind loading of large telescopes," in Large Ground-Based Telescopes, J. M. Oschman and L. M. Stepp, eds., Proc. SPIE 4837, pp. 352-367 (2002).

5. G. Z. Angeli, M. K. Cho, M. Sheehan, and L. M. Stepp, "Characterization of wind loading of telescopes," in Integrated Modeling of Telescopes, T. Andersen, ed., Proc. SPIE 4757, 72-83 (2002).

6. T. Pottebaum and D. G. MacMynowski, "Buffeting of large telescopes: wind tunnel measurements of flow in a generic enclosure," J. Fluids Struct. 22, 3-19 (2006).

7. K. Vogiatzis, A. Segurson, and G. Z. Angeli, "Estimating the effect of wind loading on extremely large telescope performance using computational fluid dynamics," in Modeling and Systems Engineering for Astronomy, S. C. Craig and M. J. Cullum, eds., Proc. SPIE 5497, pp. 311-320 (2004)

8. F. Forbes and G. Gabor, "Wind loading of large astronomical telescopes," in Advanced Technology Telescopes, Proc. SPIE 332, 198-205 (1982).

9. M. Ravensbergen, "Main axes servo systems of the VLT," in Advanced Technology Optical Telescopes V, L. M. Stepp, ed., Proc. SPIE 2199, 997-1005 (1994).

10. T. M. Erm and G. Z. Angeli, "TMT wind model validation with measurements at Keck and Gemini," in Modeling, Systems Engineering and Program Management for Astronomy II, M. Cullum and G. Angeli, eds., Proc. SPIE 6271, 62710N (2006).

11. T. Kiceniuk and K. Potter, Internal Air Flow Patterns for the
Keck 10 Meter Telescope Observatory Dome, Keck Observatory Report \#166 (Keck Observatory, 1986).

12. M. Schneermann, "VLT enclosures wind tunnel tests and fluid dynamic analyses," in Advanced Technology Optical Telescopes V, L. M. Stepp, ed., Proc. SPIE 2199, 465-476 (1994).

13. J. Fitzsimmons, J. Dunn, G. Herriot, L. Jolissaint, S. Roberts, M. Mamou, and K. Cooper, "Predicting the aerodynamic performance of the Canadian very large optical telescope," in Modeling and Systems Engineering for Astronomy, S. C. Craig and M. J. Cullum, eds., Proc. SPIE 5497, 321-328 (2004).

14. H. Riewaldt, M. Lastiwka, N. Quinlan, K. McNamara, X. Wang, T. Andersen, and A. Shearer, "Wind on the Euro50 enclosure," in Astronomical Structures and Mechanisms Technology, J. Antebi and D. Lemke, eds., Proc. SPIE 5495, 537548 (2004)

15. D. S. DeYoung, "Numerical simulations of airflow in telescope enclosures," Astron. J. 112, 2896-2908 (1996).

16. S. Padin, "Wind-induced deformations in a segmented mirror," Appl. Opt. 41, 2381-2389 (2002).

17. K. Vogiatzis and G. Z. Angeli, "Strategies for estimating mirror and dome seeing for TMT," in Modeling, Systems Engineering and Program Management for Astronomy II, M. Cullum and G. Angeli, eds., Proc. SPIE 6271, 627100 (2006).

18. J. E. Rossiter, "Wind-tunnel experiments on the flow over rectangular cavities at subsonic and transonic speeds," Aeronautical Research Council Reports and Memoranda, No. 3438, (1964).

19. D. G. MacMartin, "Control challenges for extremely large telescopes," in Smart Structures and Materials 2003: Industrial and Commercial Applications of Smart Structures Technology, E. H. Anderson, ed., Proc. SPIE 5054, 275-286 (2003).

20. V. Yakhot, S. A. Orszag, S. Tangham, T. B. Gatski, and C. G. Speziale, "Development of turbulence models for shear flows by a double expansion technique," Phys. Fluids A 4, 15101520 (1992).

21. B. E. Launder and D. B. Spalding, "The numerical computation of turbulent flows," Comput. Methods Appl. Mech. Eng. 3, 269-289 (1974).

22. J. Smagorinsky, "General circulation experiments with the primitive equations: 1 . the basic experiment," Mon. Weather Rev. 91, 99-164 (1963).

23. W. R. Sears, "Some aspects of non-stationary airfoil theory and its applications," J. Aeronaut. Sci. 8, 104-108 (1941).

24. H. W. Liepmann, "On the application of statistical concepts to the buffeting problem," J. Aeronaut. Sci. 19, 793-800 and 822 (1952).

25. A. G. Davenport, "Buffeting of a suspension bridge by storm winds," J. Struct. Div. ASCE 88, 233-264 (1962).

26. G. R. Srinivasan, "Acoustics and unsteady flow of telescope cavity in an airplane," J. Aircr. 27, 274-281 (2000).

27. D. Rockwell, J.-C. Lin, P. Oshkai, M. Reiss, and M. Pollack, "Shallow cavity flow tone experiments: onset of locked-on states," J. Fluids Struct. 17, 381-414 (2003).

28. C. W. Rowley, D. R. Williams, T. Colonius, R. M. Murray, and D. G. MacMynowski, "Linear models for control of cavity flow oscillations,” J. Fluid Mech. 547, 317-330 (2006).

29. K. Karbon and R. Singh, "Simulation and design of automobile sunroof buffeting noise control," in Eighth AIAA / CEAS Aeroacoustics Conference, June 2002, AIAA 2002-2550 (AIAA, 2002). 